it can, for example, be mounted on a mobile support. Moreover, the intensity of the radiation produced is greater than with the customary betatrons, for several reasons : because of the high frequency, the energygain in each loop is considerable (240 volts); this effects strong damping of the oscillations about the equilibrium orbit, while, moreover, the total path of the electrons is not more than $30 \mathrm{~km}$. Consequently the loss of electrons by impact against the wall or against the molecules of the residual gas is considerably smaller than at the lower frequencies commonly used. In addition, a greater number of electrons can be accelerated at a time, owing to the large dimensions of the acceleration tube. Lastly, the axial symmetry of the magnetic field is more perfect than in an apparatus with pole pieces arranged in sectors, which again reduces the loss of electrons. Reliable data on the intensity of the radiation are not as yet available, but our experiments have already yielded evidence that the peak intensity is much higher than that obtained, in this laboratory, with a betatron of the customary type.

\section{MULTIPLICATION AND DIVISION BY ELECTRONIC-ANALOGUE METHODS}

\author{
By E. M. DEELEY and D. M. MACKAY \\ Wheatstone Laboratory, King's College, London
}

$\mathrm{T}$ HE product or quotient of two rapidly varying quantities is difficult to obtain by conventional electronie-analogue methods. Desirable features in a multiptier include: $(a)$ symmetry of response to positive and negative inputs; $(b)$ an absolute indication of zero input, with consequent freedom from zero drift; $(c)$ independence of normal changes in electponic characteristics ; $(d)$ rapidity and accuracy of response.

Techniques such as amplitude-, frequency- or pulse-modulation adapted from the field of communications have so far failed to provide all these features ; most are fundamentally incapable of doing so. The object of this note is to direct attention to a simple principle which has been found to meet the above requirements ${ }^{1}$, and which seems to invite wider application in this and related fields.

It is most easily described as a development of the principle used in J. J. Thomson's familiar method of measuring electron velocity, in which the force on an electron moving with velocity $v$ at right-angles to a magnetic field $H$ is counteracted by an electrostatic field $E$ proportional to the product $H \times v$. If the adjustment of $E$ were automatic and instantaneous, its value would be a continuous measure of this product.

The cathode-ray tube, which is well known to possess potentially all four virtues above, forms a convenient basis for experimental work. In Thomson's arrangement, however, $v$ was the axial velocity of the electron stream, which is not a widely variable or reversible quantity. Accordingly, in the practical form of multiplier shown in essentials in the accom. panying diagram, an axial magnetic field is used and $v$ is the transverse velocity of the electron stream in the $X$-direction, proportional to a voltage $V_{x}$ applied to conventional deflecting plates $X_{1}, X_{2}$. After $X$-deflexion, the stream passes through the magnetic field $H$, which produces a deflecting force, initially in the $Y$-direction, proportional to $H \times V_{x}$.

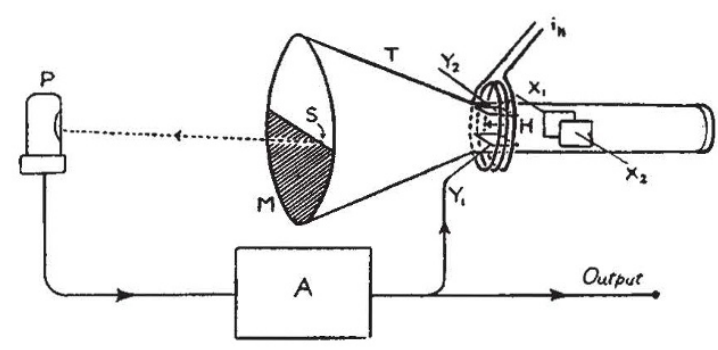

The $Y$-deflexion of the spot $S$ on the screen, which would normally result (corresponding to a rotation of the pattern), is counteracted by a photo-electric feedback system of the type used in function. generators ${ }^{2,3}$. The photocell $P$, coupled to one of the $Y$-plates via the amplifier $A$, prevents the spot from deviating by more than a fraction of its diameter from the edge of the mask $M$. With care in adjust. ment of the latter, the spot can thus be held closely to the $X$-axis, and $V_{y}$ is then proportional to the product of $V_{x}$ with the current $i_{H}$ producing $H$. Conversely, if the photocell is used to control $H$ and voltages $V_{x}, V_{y}$ are applied to $X$ and $Y$ plates, the current $i_{H}$ is proportional to the quotient $V_{y} / V_{x}$.

Since the important parameters other than the accelerating voltage are purely geometrical, the prospects of attaining high stability in a device of this kind are good. Output is sensibly independent of changes in the gain of $A$, as usual in feedback circuits. At a later stage, it is hoped to dispense with the photo-electric link, using a collecting. electrode inside the tube to perform the combined function of cell and mask.

At the time of writing, it has not been possible to make a fair test of the accuracy and frequency-range of the technique; but it may be of interest to sum. marize the encouraging results of preliminary experiments with quite crude apparatus. A 931-A photomultiplier has been used, with a cathode-ray tube having a blue screen and running at only $500 \mathrm{~V}$. to enhance overall sensitivity; D.c. and sinusoidal inputs up to $10 \mathrm{kc}$. $/ \mathrm{sec}$. have been multiplied over the ranges $\pm 300 \mathrm{~mA}$. and $\pm 20 \mathrm{~V}$., with an accuracy of the order of 2 per cent at $50 \mathrm{c} . / \mathrm{sec}$.

Accuracy is limited in practice fundamentally by the signal to noise ratio attainable in the feedback path. Other important factors are the uniformity of the axial field, and its effective axial length, which should not greatly exceed that of the $Y$-plates. If magnetic $X$-deflexion were used, the $Y$-voltage would be practically independent of 'gun' voltage; but if electrostatic $X$-plates are employed for the sake of their high impedance, the output is directly proportional to the square root of the gun voltage, the stability of which is then an important factor in overall accuracy.

The importance of such devices to the physicist and mathematician lies chiefly in their application to the solution by analogue methods of differential equations with non-constant coefficients. A number of further applications of the same principle to highspeed electronic computing technique are being developed, and a fuller account of these and the above will be published elsewhere.

\footnotetext{
${ }^{1}$ MacKay, D. M., Brit. Prov. Pat. Spec. No. 11161 (1948). The same principle has been the subject of an unpublished independent investigation by D. J. Mynall.

${ }^{2}$ MacKay, D. M., Nature, 159, 406 (1946).
}

${ }^{3}$ Mynall, D. J., Nature, 159, 743 (1946). 